\title{
Template-Free Parallel One-Dimensional Assembly of Gold Nanoparticles
}

\author{
Yu Xin Zhang, Hua Chun Zeng* \\ Department of Chemical and Biomolecular Engineering, Faculty of Engineering \\ National University of Singapore, 10 Kent Ridge Crescent, Singapore 119260 \\ *Tel: (65) 6516-2896, Fax: (65) 6779-1936, Email: chezhc@nus.edu.sg
}




\section{SI-1 STEM and FESEM measurements}

The following is a pair of complementary STEM and FESEM images measured for an identical sample area, which shows that there is no special feature of the pristine TEM sample grid that contributes to the observed assemblies. However, due to the small Au nanoparticles and surface charging, the Au lines observed in STEM image cannot be detected in FESEM image. Nevertheless, the structural effect of TEM sample grid on the observed assemblies can be ruled out. Light blue arrowed lines are to guide your comparison.

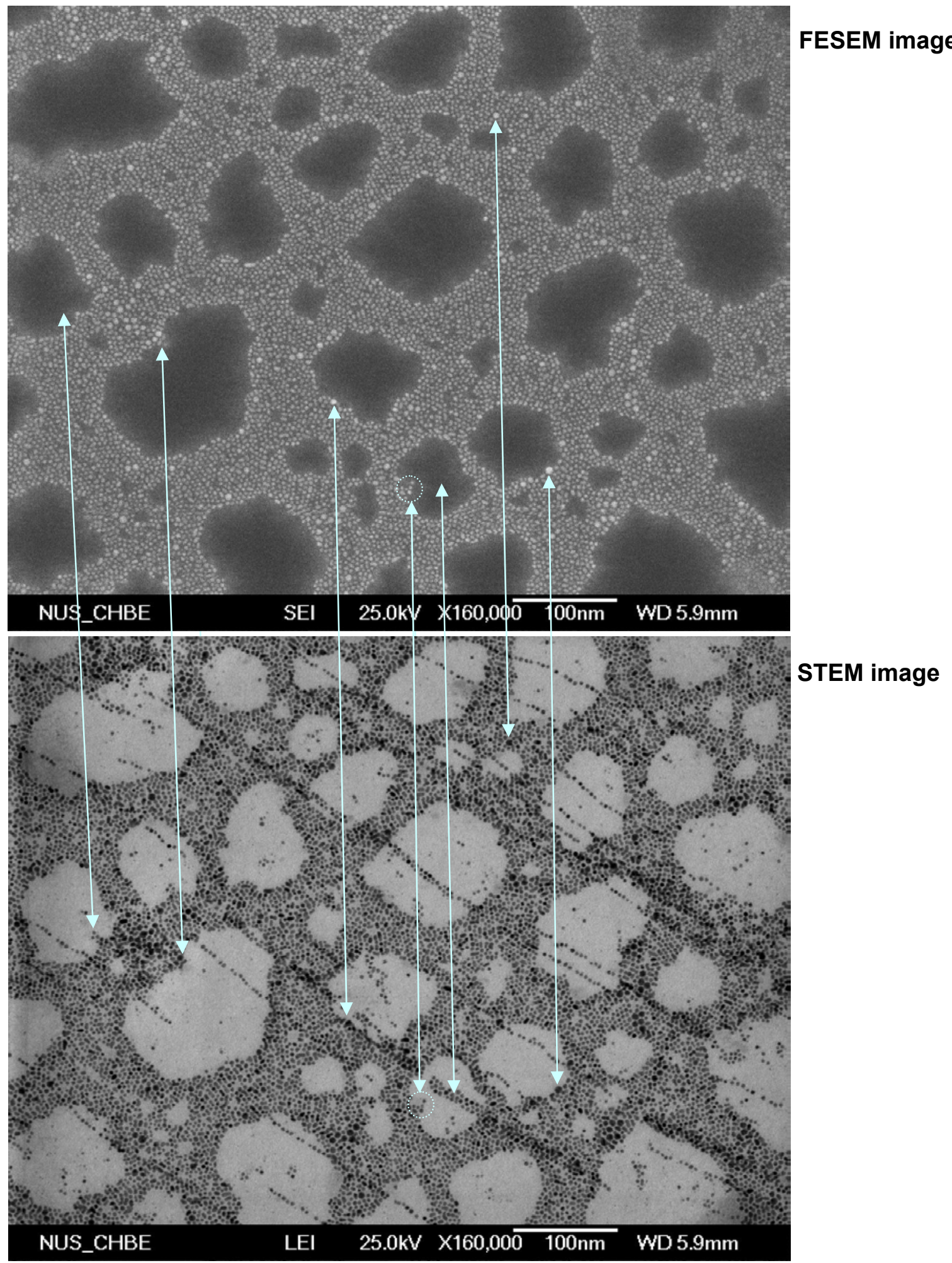


SI-2 Detailed FTIR spectra over the $\mathrm{C}-\mathrm{H}$ vibrational region

The following representative FTIR spectra show detailed peaks of $\mathrm{C}-\mathrm{H}$ vibrational modes described in the main text for the as-prepared, ethanol-washed, and heated Au nanoparticles (refer to Figure $3 a$ of the main text for the overall spectra).

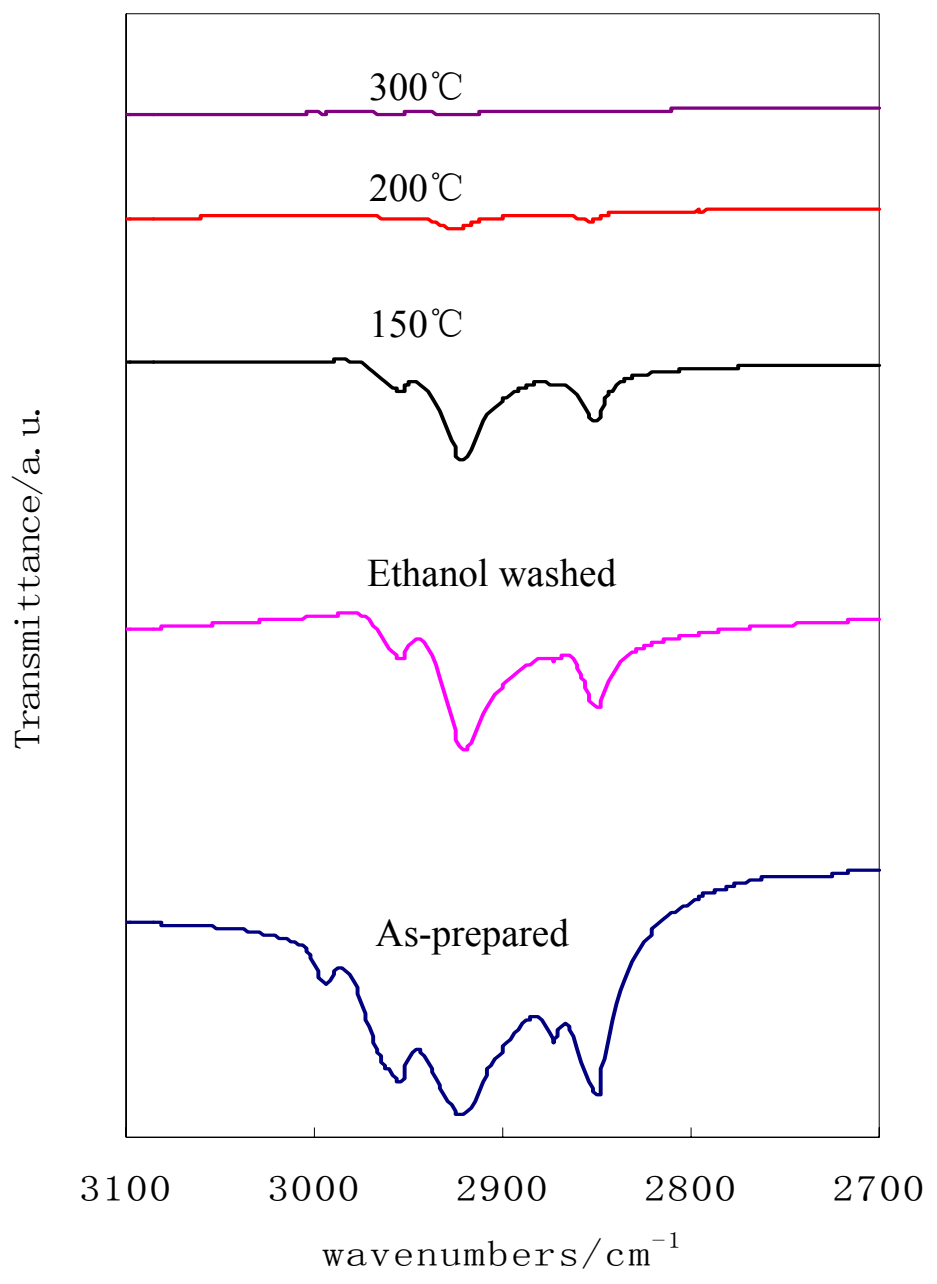


SI-3 One-dimensional assembly of the washed sample (without heating)

The following three representative TEM images reveal that the 1D-assembly of Au nanoparticles can still be attained for small-sized Au nanoparticles (without heat-treatments) after washed with ethanol (i.e., the ethanol-washed sample), although the population of these chains is not as high as those shown in Figure $1 \mathrm{a}, \mathrm{b}$ of the main text.
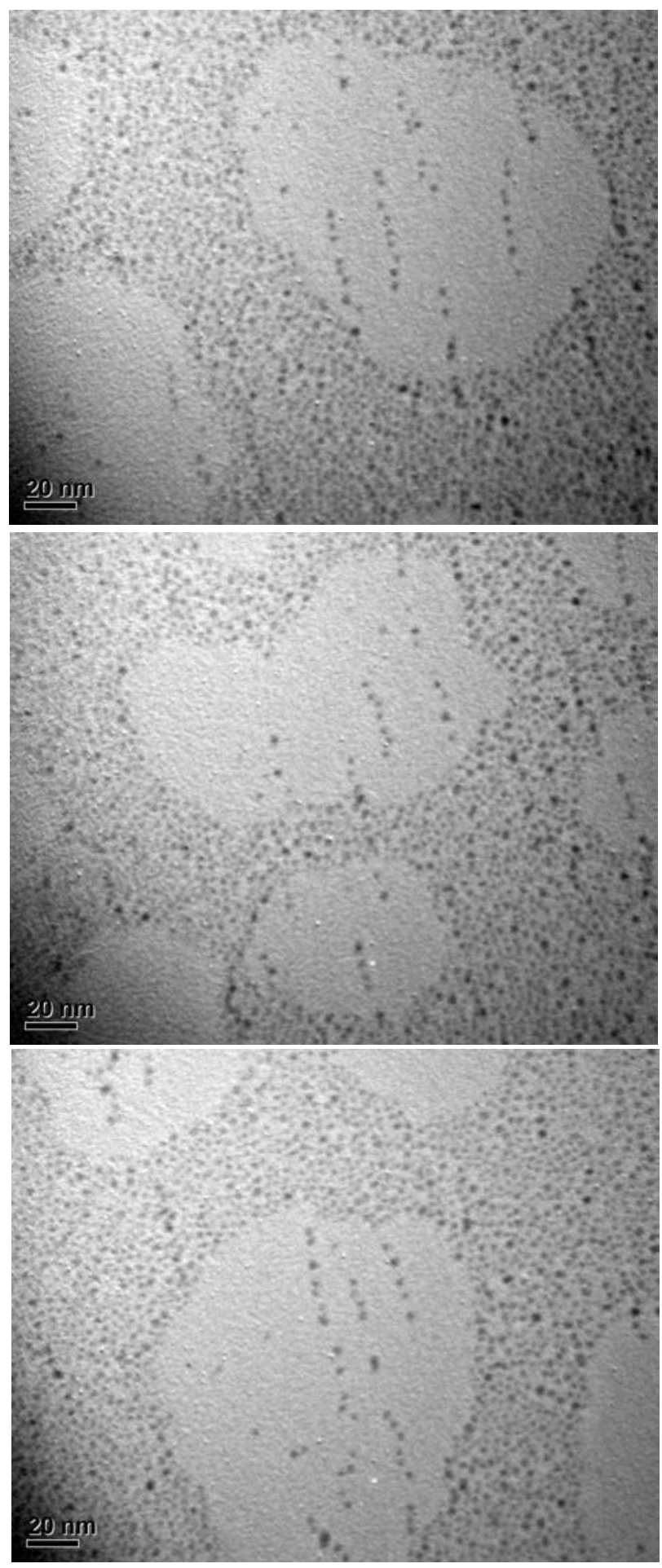